\title{
Validation of Heat Conduction 2D Analytical Model in Spherical Geometries using infrared Thermography. *
}

\author{
by C. San Martín ${ }^{1,2}$, C. Torres ${ }^{1,2}$, D. Esparza 2 , D. Bonilla ${ }^{2}$, R. Donoso ${ }^{1,2}$. \\ Center for Optics and Photonics CEFOP, University of La Frontera, Chile ${ }^{1}$. \\ Information Processing Laboratory IPL, University of La Frontera, Casilla 54-D, Temuco, Chile ${ }^{2}$. \\ cesarsanmartin@ufro.cl
}

\begin{abstract}
This work presents an experimental validation of an analytical model of heat conduction in spherical samples using infrared thermography. The theoretical model is a two dimensional analytical solution of the heat equation considering a radiant heating source. This model describes the physical behavior of the heat conduction in twodimensional solid objects considering spherical geometries. The model dependent on the radius and angle values and this is fit with the measured data using a thermal camera. A synthetic image obtained using this model is compared with the values obtained from a camera, for spherical geometry presenting good agreement.
\end{abstract}

Keywords: Heat Conduction 2D, Infrared Thermography and Spherical Geometry.

\section{Introduction}

The infrared image acquisition has the advantage of being a noninvasive and contactless technique. This allows that the object remains unaltered when the measuring is performed. Also, spatial-temporal information of a single or multiple objects can be obtained simultaneously. This work presents an experimental scheme based on the measurement of the infrared radiation emitted, without considering emissivity of the specimen or measuring its temperature. Infrared thermography is a technique used for the radiometric measurement of the surface temperature using infrared sensors [1,2], where the radiation and temperature are related by $I=\sigma \varepsilon_{s} T^{4}[8,6]$, where $\sigma$ is StefanBoltzmann's constant (equal to $\left.5.67044 \times 10^{-8}\left(\mathrm{~W} / \mathrm{m}^{2} \mathrm{~K}^{4}\right)\right)$ and $\varepsilon_{\mathrm{s}}$ is the emissivity of the sample. On the other hand, an analytical solution for the heat conduction equation is obtained using classical theory for spherical geometries and radiant heating process. The images from the analytical model are compared with the real infrared data, validating the analytical solutions obtained from literature.

This work is organized as follows. In section 2, the mathematical model of heat conduction is formulated. In section 3, the experiment and quantitative evaluation process is presented. Later, in section 4 the results are presented and discussed. Finally, this work ends with some conclusions in section 5.

\section{Mathematical model formulation}

We consider the continuous radiant heating of opaque spherical sample with $R(m)$ radius, $\alpha$ the thermal diffusivity $\left(\mathrm{mm}^{2} / \mathrm{s}\right), k$ the thermal conductivity $(\mathrm{W} / \mathrm{m} / \mathrm{K})$ and $\varepsilon$ the emissivity. The radiation source correspond a halogen lamp with the spectral radiation emission characteristics assumed to be known. In this case, the sample is illuminated by parallel radiation and only diffuse reflection is considered. In this paper the spatial temperature distribution is calculated in spherical coordinates assuming that the source is independent of $\Phi$, and the heat transfer equation in this case is given by [3]:

$$
\frac{\partial u}{\partial t}=\frac{\alpha}{r^{2}} \frac{\partial}{\partial r}\left(r^{2} \frac{\partial u}{\partial r}\right)+\frac{\alpha}{r^{2} \sin \theta} \frac{\partial}{\partial \theta}\left(\sin \theta \frac{\partial u}{\partial \theta}\right)
$$

where $u$ is the temperature in degrees Kelvin. The boundary conditions at the surface $r=R$ is:

$$
k \frac{\partial u}{\partial r}=h_{c} u+\sigma \varepsilon_{S} u^{4}-\frac{a \pi \varepsilon_{S} I}{2}(\cos \theta+|\cos \theta|)
$$

where $h_{c}$ is the convection coefficient $\left(W / \mathrm{m}^{2} / K\right)$, $a$ is the absorbance factor of the sample and $I$ is the intensity of radiation of the source. In order to solve the heat conduction equation the temperature is assumed only in steady state and equation (1) is modified to:

$$
\frac{\alpha}{r^{2}} \frac{\partial}{\partial r}\left(r^{2} \frac{\partial u}{\partial r}\right)+\frac{\alpha}{r^{2} \sin \theta} \frac{\partial}{\partial \theta}\left(\sin \theta \frac{\partial u}{\partial \theta}\right)=0,
$$

This work was partially supported by Center for Optics and Photonics FB0824/2008. 
and the boundary conditions (2) must be satisfied. The solution for this equation is [4]:

$$
u(r, \theta)=\sum_{n=0}^{\infty} A_{n}\left(\frac{r}{R}\right)^{n} P_{n}(\cos \theta)
$$

where $A_{n}$ are obtained using (2) resulting [4]:

$$
\begin{gathered}
A_{n}=(2 n+1) \frac{a \pi \varepsilon_{S} I R}{4\left[k n+h_{T} R\right]}\left[\frac{2}{(2 n+1)}\left[\frac{(n+1)}{(n+2)} P_{n}(0)+P_{n-2}(0)\right]\right] \\
A_{n}=\frac{a \pi \varepsilon_{S} I R}{2\left[k n+h_{T} R\right]}\left[\frac{(n+1)}{(n+2)} P_{n}(0)+P_{n-2}(0)\right]
\end{gathered}
$$

\section{Experiment and Quantitative Assessment}

The model given by (4) is simulated using a 3D visualization system based on ray tracing theory. In this case, we generate the model varying the angle on the surface $(r=R)$. On the other hand, the sample used corresponds to foam which has a radius of $5 \mathrm{~cm}$. Foam is a material which diffuses reflection, and is more comparable with the solutions of (1) subject to (2). A FLIR Photon 320 infrared camera $(8-14 \mu \mathrm{m})$ is used to capture the infrared images in reflection mode, and a halogen lamp is used as a radiant source. The procedure used starts heating the sample. Due to the thermal parameters of foam, we wait until the temperature remains constant after 45 minutes approximately. In this case we have the steady spatial temperature. The setup is presented in Figure 1a. The analytical model generated is shown in Figure 1b. Finally, the Figure 1c presents the real infrared image from FLIR camera.
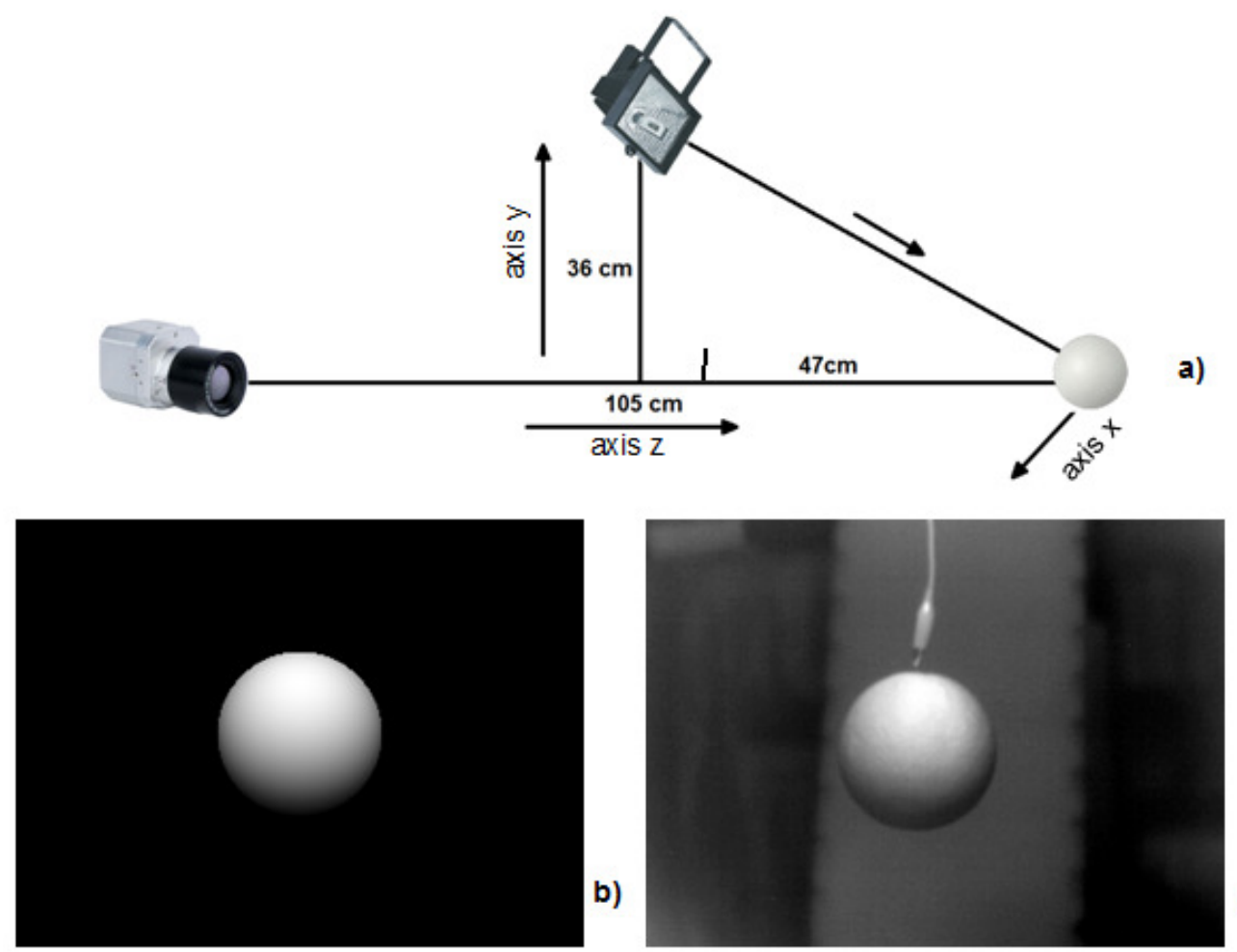

c)

Fig. 1. a) Experimental setup to obtain infrared images, b) Synthetic images using analytical model,

c) Thermal images of foam considering steady state temperature.

We aforementioned the heat conduction model given by (4) has been simulated using a 3D visualization system based on ray tracing theory. With this model we generate a synthetic image that is used to match with the image 
obtained from infrared camera (see Figure 1b). The ray tracing theory allows to reverse the process and assign to each pixel of the infrared image (obtained by the FLIR Photon 320 (see Figure 1c)) a spatial location.

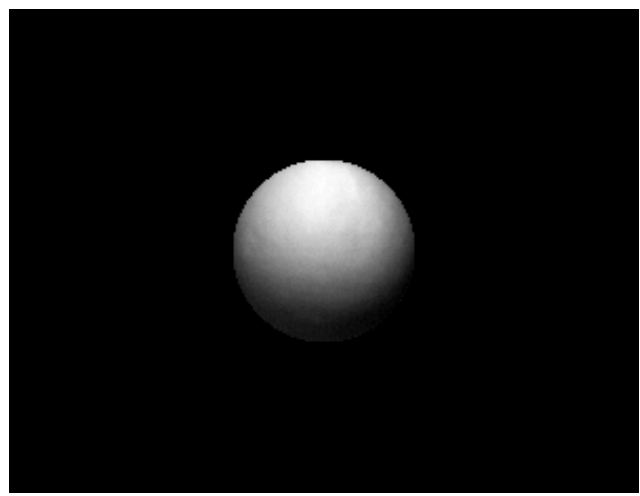

Fig. 2. Removing the foam sphere of thermal image.

The quantitative evaluation process used in this paper was to compare points belonging to the surface of spherical geometry. These points have a synthetic value of $u$ (see equation 4), given by the analytical model and a real value obtained by the 3D model reconstructed since the infrared image (see Figure 2). The points used for comparison are obtained by the intersection of a plane (or slice) with the surface of spherical geometry at a distance $d$ from the center of the sample (see Figure 3a). For each point $P_{i}$ obtained in this process a representative value is sought by the average of the points belonging to the plane $p^{\prime}$ (see Figure $3 b$ ), due to that the heat dissipation of the sample is identical in all directions, then all values for the same $\Phi$ should be identical. Finally, the representative value obtained for each point $P_{i}$ found on the surface of the sphere, is compared with the synthetic value calculated by the model, and this way the Mean Square Error (MSE) and the Mean Relative Error (MRE) are obtained.

To perform the above process, let be $v$, a unit vector which points in the same direction in which it spreads the light generated by the halogen lamp. From Figure 1a we can deduce that the vector $v$ forms an angle of approximately 142.55 degrees to the $z$ axis, which is given by the FLIR Photon 320 and the foam sphere.

As shown in Figure $3 \mathrm{a}$, the slice made to the spherical geometry is given by all points belonging to the 3D model, such that belong to the plane $p$ or all points relatively close to it (ie, are at a distance less than a certain $\varepsilon$. Particularly, in this work an $\varepsilon=10^{-3}$ was used), where $p$ is a plane parallel to $v$ and the $x$ axis (note that the $x$ axis exits perpendicularly to the figure).

For each point $P_{i}$ belongs to the slice (see Figure 3a) other slice $(p)$ is constructed, such that $P_{i} \in p^{\prime}$ and the plane $p^{\prime}$ is perpendicular to the vector $v$ (Note that in spherical coordinates, the plane $p^{\prime}$ is given by a same $\phi$ ). Then calculates the average of real values representing the points belonging to $p^{\prime}$, values obtained by the reconstructed $3 D$ model using the ray tracing theory and an image like Figure 2.
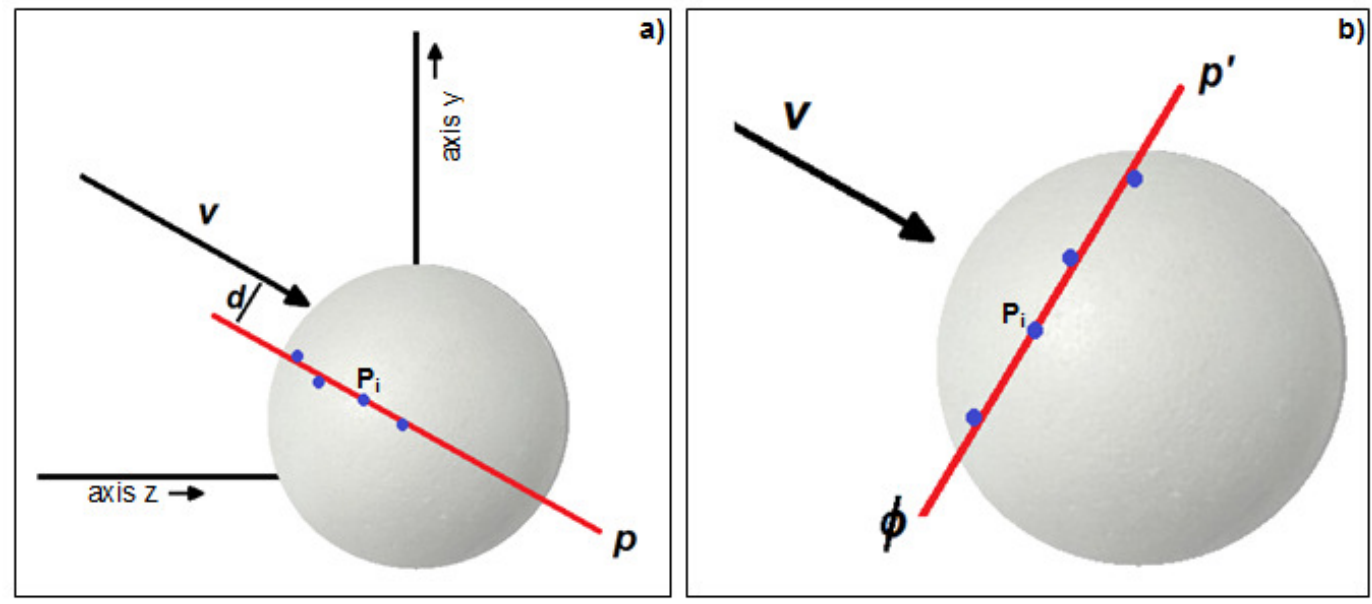

Fig. 3. a) Points describing a slice to the spherical geometry. b) Points on the plane p' described by the same $\phi$. 
To find a suitable amount of points belonging to the surface of the sphere, 4000 planes ( $p$ type) or parallel slices to $v$ are generated. Initially, $d=0$ was considered, and later the separation distance was increased in 0.001 on each occasion. With this process, about 180 points were obtained. For each point above, a plane $p$ ' is generated and its value was averaged about 4 to 6 points more.

Finally, with the 180 averaged values (extracted from the image acquired by the infrared camera (see Figure 2) using the ray tracing theory) and theirs counterpart, the values generated by the analytical model of heat conduction, the mean square error (MSE) and mean relative error (MRE) is calculated through the following formulas:

$$
\begin{aligned}
& M S E=\sqrt{\sum_{i=1}^{n} \frac{\left(\text { realValue }_{i}-\text { syntheticValue }_{i}\right)^{2}}{n}} \\
& M R E=\frac{1}{n} \sum_{i=1}^{n} \frac{\text { abs }\left(\text { realValue }_{i}-\text { syntheticValue }_{i}\right) * 100}{\text { syntheticValue }_{i}}
\end{aligned}
$$

The results and graphs obtained from this experiment are presented in the following section.

\section{Results}

Considering that $\theta$ is the angle between the normal vector to the surface of the sphere and the unit vector $v$ in the direction of light propagation (see Figure 3a). Then, as shown in Figure 4 the heat conduction model assigns values close to one when $\theta$ is close to zero, on the other hand, the model assigns values close to zero when $\theta$ is close to $\pi / 2$, i.e., the function given by Equation 4 is proportional to $\cos (\theta)$. This can be confirmed with a naked eye by analysis the model of heat conduction.
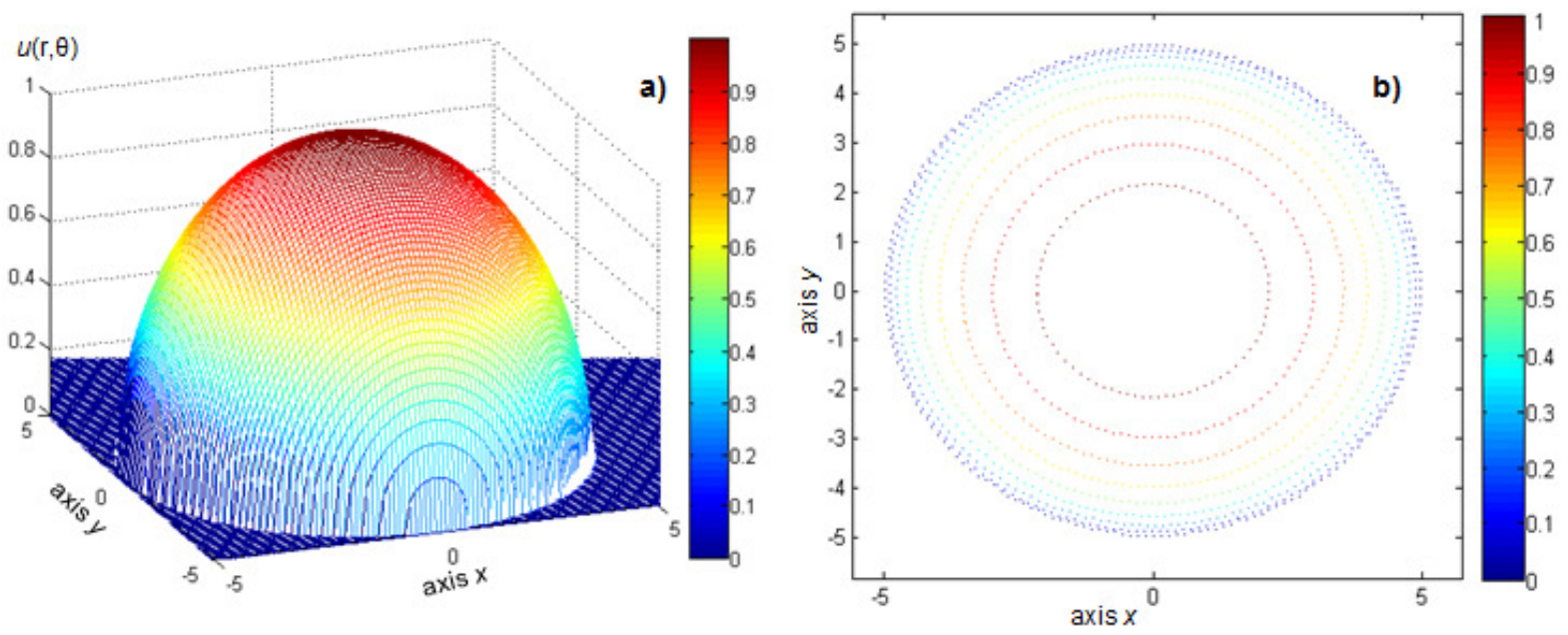

Fig. 4. a) Graphics of $3 D$ Heat Conduction Model. b) Perpendicular slices of $3 D$ model.

The Figure 5 shown that qualitatively it is possible to appreciate that the real values (represented by the red asterisks on this figure) were adjusted to heat conduction model, even though some points cannot be seen (i.e., their values are smaller than model values), and some points can be fully appreciate (i.e., their values are bigger than model values).

Note that the points that can be partially appreciated are the points that intersecting the surface of the 3D model, i.e., the real value obtained from the thermography image is very close to the synthetic value predicted by the model of heat conduction.

The most important thing that is possible to see in Figure 5, is that although there are small differences between real values and the values obtained using the analytic model, there are no outliers or points beyond the predicted behavior by the model.

Also, keep in mind that in this work the fixed pattern noise and the variable pattern noise wasn't considered. These two types of noise may be affecting the infrared image acquisition process and produce small differences between the real and synthetic image. 


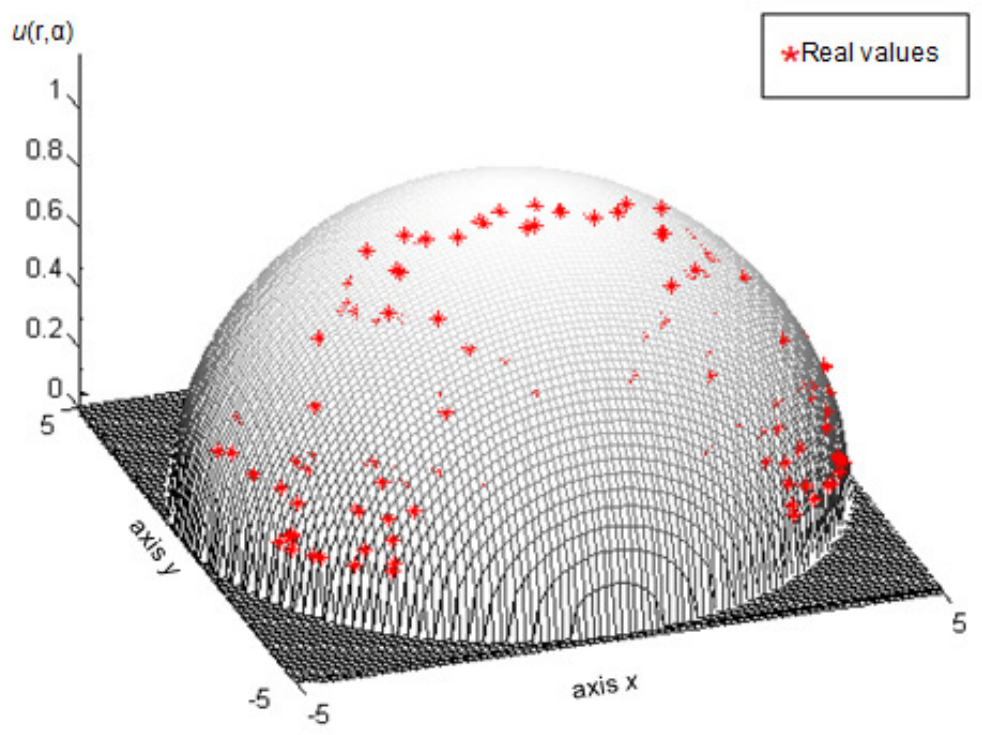

Fig. 5. Graphics of 3D Heat Conduction Model with real values points (*).

As shown in Figure 6, the values represent the 180 points on the sample surface, which are associated with different values as the $\cos (\theta)$ (represented by the blue line), the value $u(r, \theta)$ obtained by the heat conduction model (represented by the red line) and finally the real value obtained from the infrared image using the process described in section 3 (represented by the green line).

Note that the points plotted in this figure are not sorted, since it if they were sorted; they will have a very similar behavior to the cosine function (see Figure 7). This figure shows only the similarity between the curves, especially between the curve generated by the real values and the values of the heat conduction model.

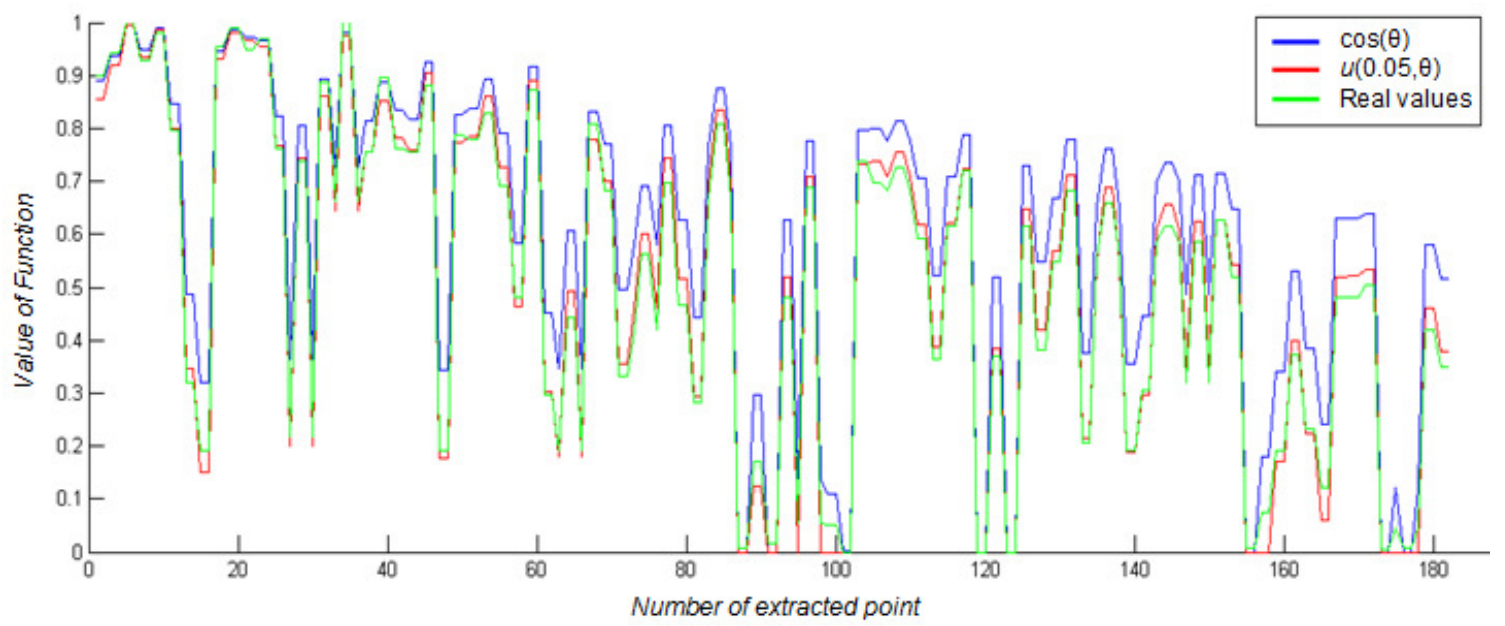

Fig. 6. Graphic of 180 points extracted since the sample surface by the method of parallel slices. This figure shows the values of cosine, of analytic model and real values for each point.

As shown in Figure 6, the curves generated by the model and real values are adjusted properly, this it's possible to confirm with the values of $\mathrm{MSE}=0.0289$ and $\mathrm{MRE}=0.0339 \%$ calculated with the equations (6) and (7) respectively. Note that the MRE value represents the average percentage change between the error and the expected data, i.e., on average an error of only $0.0339 \%$ from the expected value is done. 


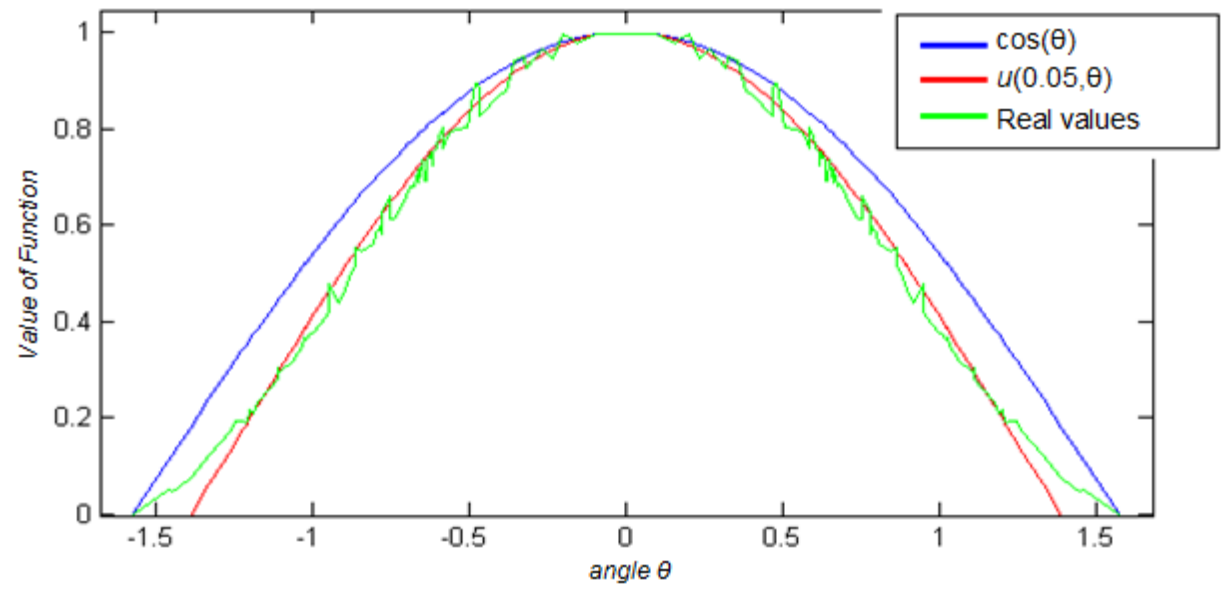

Fig. 7. Graphic of 180 points extracted since the sample surface by the method of parallel slices. This figure shows the values of cosine, of analytic model and real values for each point in an orderly way according to the angle $\theta$.

Unlike Figure 6, the Figure 7 shows the values in order, which allows to foresee the similarity of the heat conduction (both the real values as synthetics) with the function $\cos (\theta)$. Furthermore it can be seen that the heat conduction model is suitably adjusted to the real values.

\section{Conclusions}

In this work we used infrared thermography and ray tracing theory to validate a 2D analytical model for heat conduction in spherical geometries.

From Figure 6, is concluded that the heat conduction model is suitably adjusted to the real data obtained from the thermography image, which is reflected in a small mean square error of the order of $10^{-2}$, and subsequently in a mean relative error of $0.03 \%$. Whereas it was necessary to obtain these values incur an error of the order of $10^{-3}$ for finding points on the plane $p^{\prime}$ and so, find the 180 values representative of the points on the sample surface by averaging, in each case, the points belonging to $p^{\prime}$.

We also conclude that as outliers don't appear in any of the figures shown in this paper, the behavior of heat conduction model is adequate, and that small differences between real and synthetic values can be associated with the uncontrolled noises of thermal imaging.

For all these reasons we conclude that the experiment conducted by the ray tracing theory and infrared thermography, definitely validates the $2 \mathrm{D}$ analytical model for heat conduction in spherical geometry, since it with a naked eye the behavior of analytic model is adequately fit the curve of the real data, which is confirmed by the reduced values of MSE and MRE obtained.

Our study will be extended by validating other transient temperatures in different materials. For materials with specular reflections it is necessary to add some terms to the equations (2).

\section{Acknowledgements}

The authors wish to thank the Center of Optics and Photonics (CEFOP), at the "University of Concepción", Concepción, Chile. Also, the authors wish to thanks to Information Processing Laboratory (IPL), at the "University of La Frontera", in Temuco, Chile.

\section{REFERENCES}

[1] Maldague, X. "Theory and Practice of Infrared Technology for Nondestructive Testing", 2ª Ed., John Wiley \& Sons, Inc., New York, 2001.

[2] Maldague, X. "Introduction to NDT by Active Infrared Thermography". Materials Evaluation, 2002.

[3] Carslaw, H.S., Jaeger, J.C. "Conduction of Heat in Solids", 2 ${ }^{\mathrm{a}}$ Ed., Oxford University Press, London, 1959.

[4] M. R. Thorpe, "Radiant Heating of Apples". Journal of Applied Ecology, Vol. 11, No. 2 (1974), pp. 755-760. 
[5] H. Bateman, "Partial differential equations on mathematical physics", Dover Publications, 1944.

[6] L. Boltzmann, "Ableitung des stefan'schen gesetzes, betreffend die abhängigkeit der wärmestrahlung von der temperatur aus der electromagnetischen lichttheorie", Wiedemann's Annalen, Bd. 22 (1884), pp. 291-294.

[7] H. S. Carslaw, "Heat and Mass Transfer between Impinging Gas Jets and Solid Surfaces", Advances in Heat Transfer, Vol. 13 (1977), pp. 1-60, Academic Press, New York.

[8] J. Stefan, "Über die Beziehung zwischen der Wärmestrahlung und der Temperatur, in: Sitzungsberichte der mathematisch-naturwissenschaftlichen Classe der kaiserlichen Akademie der Wissenschaften”, Wiener Ber ,Bd. 79 (1879), pp. 391-428. 